\title{
Synthesis and Characterization of an Amphiphilic Linoleic Acid-g-Quaternary Chitosan with Low Toxicity
}

\author{
Xiaobin Fang, ${ }^{1}$ Yingqi Xu, ${ }^{1}$ Jinming Zhang, ${ }^{1}$ Xianghong Lu, ${ }^{2}$ \\ Yitao Wang, ${ }^{1}$ and Meiwan Chen ${ }^{1}$ \\ ${ }^{1}$ State Key Laboratory of Quality Research in Chinese Medicine, Institute of Chinese Medical Sciences, University of Macau, Macau \\ ${ }^{2}$ Translation Medicine Research Center, Lishui People's Hospital, The Sixth Affiliated Hospital, Wenzhou Medical University, \\ Lishui, Zhejiang 323000, China
}

Correspondence should be addressed to Meiwan Chen; mwchen@umac.mo

Received 27 May 2015; Revised 20 July 2015; Accepted 26 July 2015

Academic Editor: Jin-Ho Choy

Copyright (C) 2015 Xiaobin Fang et al. This is an open access article distributed under the Creative Commons Attribution License, which permits unrestricted use, distribution, and reproduction in any medium, provided the original work is properly cited.

\begin{abstract}
A novel amphiphilic derivative of chitosan, namely, a linoleic acid-g-quaternary chitosan (LA-g-QC), was designed and synthesized as low toxic material for biomedical applications in this study. The chemical structure of LA-g-QC was characterized by Fourier transform infrared spectroscopy (FTIR), ${ }^{1} \mathrm{H}$ nuclear magnetic resonance $\left({ }^{1} \mathrm{H}-\mathrm{NMR}\right)$, and elemental analysis. LA-g-QC could form nanosized micelles with self-assembly, which was confirmed by the results of critical micelle concentration (CMC) via fluorescence spectroscopy. The average size of LA-g-QC was $140 \mathrm{~nm}$ and its zeta potential was approximately $+35.50 \mathrm{mV}$. CMC value was $31.00 \mathrm{mg} / \mathrm{mL}$. Furthermore, LA-g-QC micelles, at final concentrations between $0.94 \mu \mathrm{g} / \mathrm{mL}$ and $30 \mu \mathrm{g} / \mathrm{mL}$, did not inhibit the proliferation of HepG2 or SMMC 7721 cell lines. Taken together, LA-g-QC has low cytotoxicity and high potential for the preparation of novel drug-delivery micelles.
\end{abstract}

\section{Introduction}

Chitosan is a natural polysaccharide with $\beta$-(1-4)-linked D-glucosamine (deacetylated unit) and N-acetyl-D-glucosamine (acetylated unit) [1, 2] and the only kind of natural edible animal fibers containing free amino group alkaline cation, which is composed after the formation of deacetylation under alkaline conditions. Its molecular weight varies from a few hundred thousand to several million, depending upon different preparation conditions and the degree of deacetylation ranging from 60 to $100 \%$ [3]. Chitosan has many unique properties such as cell affinity and biocompatibility [4] and is known as the sixth element of life. Thus it can be used for the prevention and treatment of some human diseases [5]. Owing to the special molecular structure and physicochemical properties, chitosan has antimicrobial, anti-inflammatory, hemostatic, wound healing, and other bioactivities, which make it possible to be widely used in food industry, chemicals, agriculture, and bioengineering $[6,7]$.
However, chitosan is soluble neither in water nor in common organic solvents such as dilute sulfuric acid and phosphoric acid; it is only soluble in dilute hydrochloric acid, nitric acid, and most other organic and inorganic acids [8]. In the media where $\mathrm{pH}$ is above 6 , the amines of chitosan become deprotonated and, therefore, it becomes insoluble. Compared with chitin, chitosan exhibits better dissolution ability significantly contributing to its hydrophilicity and amino functional group [9]. Nevertheless, it can only dissolve in dilute acid solution, which limits its application to a large extent. Therefore, to improve the solubility of chitosan, especially solubility in water, is one of the most important modifications of chitosan [10]. Prepared by chemical modification, a large number of hydroxyl groups and amino groups of the chitosan molecular chains can be introduced into various derivatives which have different functional groups on the repeating units. Thus, the chemical modification of chitosan in chitin chemistry is one of the most active areas of research. For instance, Chen et al. [11] introduced deoxycholic 

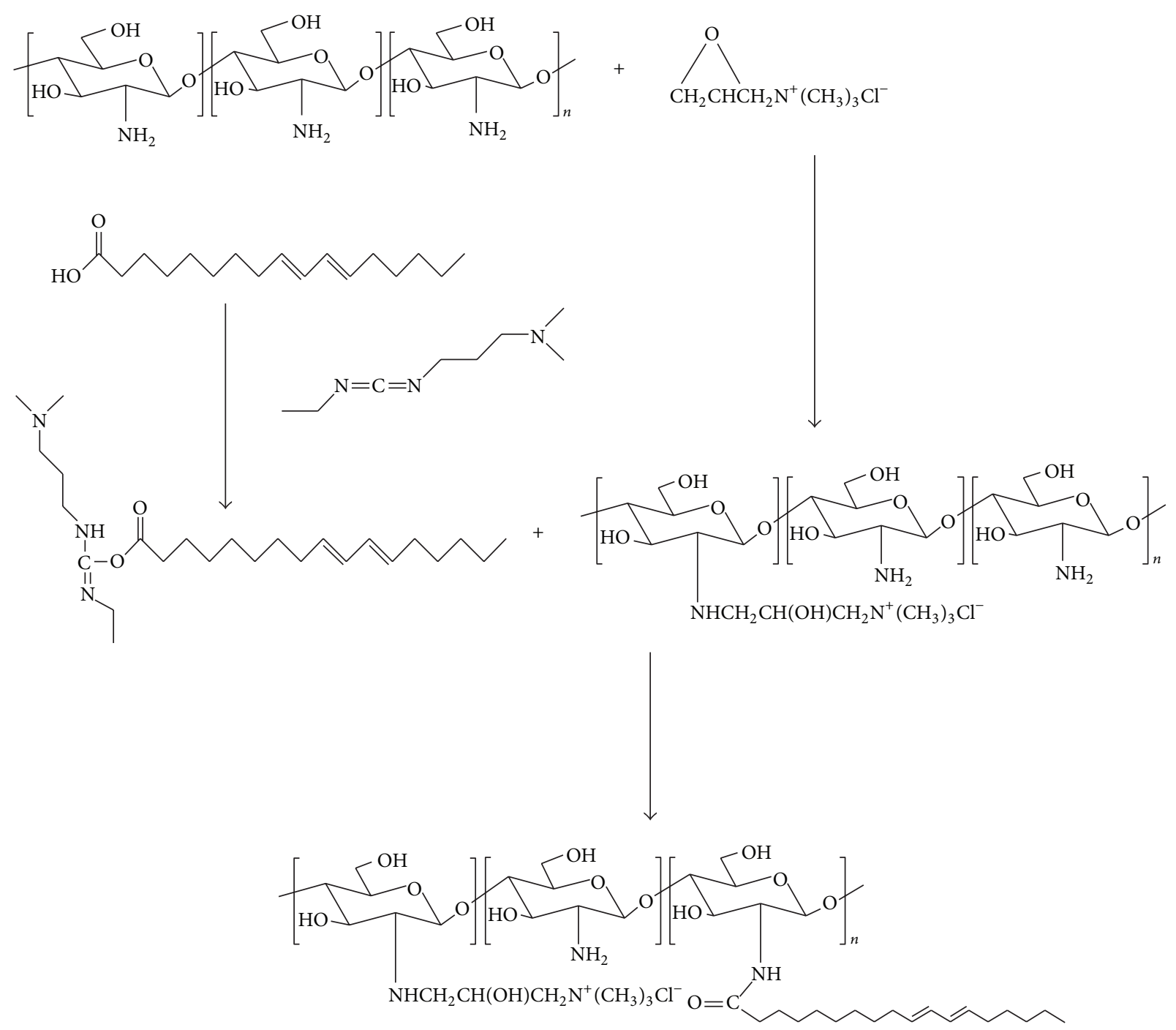

FIGURE 1: The synthetic route of LA-g-QC copolymer. The first step was the chemical reaction of ETA and the chitosan and finally the obtaining of QCS. The second step was to graft LA on QCS.

acid into chitosan, which acted as hydrophobic group, to endow chitosan with amphiphilicity.

Linoleic acid (LA), as one of the polyunsaturated fatty acids, can be easily inserted into the phospholipid bilayer of cell membrane and disorder the structure and fluidity of cell membrane, thus being rapidly taken up by cells [12]. Furthermore, it can also affect the tumor cell chemosensitivity [13]. Therefore, LA was chosen and used as hydrophobic part to modify chitosan in our research.

In this study, with an aim to improve the solubility of chitosan, an amphiphilic LA-g-quaternary chitosan (LA-g-QC) copolymer was designed and synthesized. Having confirmed its structure by ${ }^{1} \mathrm{H}-\mathrm{NMR}$, FTIR, and TEM, we fabricated LA-g-QC copolymer into micelles by using the self-assembly method and analyzed its physicochemical properties of LAg-QC micelles including CMC, particle size, zeta potential, and TEM. Furthermore, the in vitro cytotoxicity of LA-g-QC micelles was evaluated.

\section{Materials and Methods}

2.1. Materials. Chitosan $\left(M_{w} 2.0 \times 10^{5}\right.$, DD (degree of deacetylation) 85\%) was obtained from Jinan Haidebei Marine Bioengineering Co., Ltd. (Shandong, China). 2,3Epoxypropyltrimethylammonium chloride [14] was provided by Dongying Guofeng Fine Chemical Co., Ltd. (Shandong, China). LA was purchased from Aladdin Industrial Corporation (Shanghai, China). All other agents used were of analytical grades.

Human hepatoma cell lines HepG2 and SMMC 7721 were supplied by American Type Culture Collection (ATCC, Rockville, MD, USA). Fetal bovine serum (FBS), Dulbecco's Modified Eagle Medium (DMEM), and 3-(4,5-dimethylthiazol-2-yl)-2,5-diphenyltetrazolium bromide (MTT) were obtained from Sigma-Aldrich.

2.2. Preparation of $L A-g-Q C$. The LA-g-QC copolymer was synthesized by two steps as shown in Figure 1. The 
first step is obtaining quaternized chitosan (QCS) by 2,3-Epoxypropyltrimethylammonium chloride [14] under microwave radiation [15]. A certain amount of chitosan was dispersed in isopropyl alcohol, and the suspension was regulated to $\mathrm{pH} 9$ with sodium hydroxide solution. ETA aqueous solution was then added to chitosan suspension drop by drop. The whole reaction mixture was exposed to microwave irradiation at $400 \mathrm{~W}$ for $70 \mathrm{~min}$. Subsequently, reaction mixture was precipitated by the cold acetone and washed with phosphate buffer solution for three times. The second step is grafting LA on QCS. QCS, LA, and EDC were dissolved in dimethyl formamide with molar ratio $1: 1: 1.5$ under stirring for $12 \mathrm{~h}$. The reaction mixture was precipitated and washed by acetone for three times. Finally, LA-g-QC was obtained by lyophilizing.

2.3. Preparation of LA-g-QC Micelles. The LA-g-QC micelles were prepared by the solvent evaporation method. In brief, LA-g-QC copolymer $(10 \mathrm{mg})$ dispersed in the $4 \%$ ethanol solution under stirring. After stirring for $2 \mathrm{~h}$, the suspension was filtered with $0.45 \mu \mathrm{m}$ filter membrane to obtain LA-g-QC micelles solution.

2.4. Fourier Transform Infrared Spectroscopy (FTIR). Chitosan and LA-g-QC were mixed with $\mathrm{KBr}$, and the FTIR spectra of which were conducted on a NEXUS 670 FT-IR spectrophotometer (Nicolet, USA) at room temperature at $25^{\circ} \mathrm{C}$ [16]. The samples were scanned from 400 to $4000 \mathrm{~cm}^{-1}$ with a resolution of $4 \mathrm{~cm}^{-1}$.

2.5. Proton Nuclear Magnetic Resonance ( $\left.{ }^{1} \mathrm{H}-\mathrm{NMR}\right)$. The ${ }^{1} \mathrm{H}-$ NMR spectra of chitosan and LA-g-QC were characterized using liquid nuclear magnetic resonance spectrometer (400 MHz, Bruker, Germany), with $\mathrm{CF}_{3} \mathrm{COOD} / \mathrm{D}_{2} \mathrm{O}$ as solvents.

2.6. Elemental Analysis. The substitution degree (SD) of QCS and LA-g-QC was determined by elemental analysis using elemental analyzer Vario EL cube (Elementar, Germany). SD was calculated according to the content of $\mathrm{C}, \mathrm{O}$, and $\mathrm{N}$.

2.7. Transmission Electron Microscopy (TEM). LA-g-QC micelle solution was placed on a carbon-coated copper grid, observed subsequently, and photographed using high resolution transmission electron microscopy (HRTEM, Tecnai G20, FEI Company, USA) with an operating voltage of $200 \mathrm{kV}$.

2.8. Particle Size and Zeta Potential. Particle size and zeta potential of LA-g-QC micelles were measured using a dynamic light scattering Zetasizer (DLS) at $25^{\circ} \mathrm{C}$ with Zetasizer Nano ZSP System (Malvern Instruments, Worcestershire, UK).

2.9. Critical Micelle Concentration (CMC). The CMC of LA-g-QC was determined by Fluorescence Spectrometer (LUMINA, Thermo Scientific, USA) with pyrene as a hydrophobic probe. Aliquots of pyrene in acetone

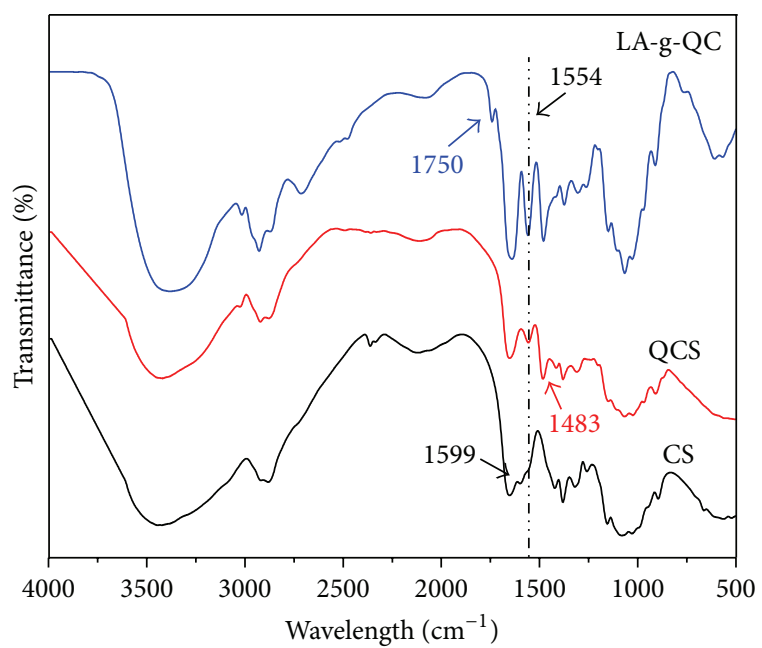

FIGURE 2: FTIR spectra of chitosan, QCS, and LA-g-QC.

$\left(6.0 \times 10^{-6} \mathrm{M}, 1 \mathrm{~mL}\right)$ were added to each vial $(10 \mathrm{~mL})$. After acetone evaporation was complete, $10 \mathrm{~mL}$ of a series of concentrations of LA-g-QC $\left(1.0 \times 10^{-4}, 1.0 \times 10^{-3}, 2.0 \times\right.$ $10^{-3}, 5.0 \times 10^{-2}, 1.0 \times 10^{-1}$, and $1.0 \mathrm{mg} / \mathrm{mL}$ ) was added to the above vials. The fluorescence emission spectra were recorded at an excitation wavelength of $339 \mathrm{~nm}$, and the emission wavelength was between 360 and $450 \mathrm{~nm}$.

2.10. In Vitro Cytotoxicity Assays. HepG2 and SMMC 7721 cells were cultivated in DMEM medium added with $10 \%$ FBS, $100 \mathrm{U} / \mathrm{mL}$ penicillin, and $100 \mu \mathrm{g} / \mathrm{mL}$ streptomycin at $37^{\circ} \mathrm{C}$ and $5 \% \mathrm{CO}_{2}$. The cytotoxicity of LA-g-QC micelles on these two cells was investigated by MTT assay [17]. The cells were seeded into 96-well plates at 6000 cells per well in $100 \mu \mathrm{L}$ of culture medium. After $24 \mathrm{~h}$ incubation, the medium was removed and replaced with medium containing various concentrations of blank polymer micelles. After the additional $24 \mathrm{~h}$ and $48 \mathrm{~h}$ incubation at $37^{\circ} \mathrm{C}$, respectively, medium without FBS containing MTT dye $5 \mathrm{mg} / \mathrm{mL}$ took place of the previous culture medium for another $4 \mathrm{~h}$ to form formazan crystals by mitochondrial dehydrogenases. Subsequently, $100 \mu \mathrm{L}$ of DMSO was supplemented to dissolve the formazan crystals in each well. The absorbance of cells was evaluated at $570 \mathrm{~nm}$ with untreated cells as controls. The absorbance ratio of treated cells and control cells was expressed as cell viability to characterize the cytotoxicity of polymers.

\section{Results and Discussion}

3.1. Synthesis and Structural Characterization of LA-g-QC. A novel amphiphilic LA-g-QC copolymer was synthesized according to the schemes shown in Figures 2 and 3. It can be seen from the FTIR spectra of chitosan that the characteristic peak lying at $1599 \mathrm{~cm}^{-1}$ attributed to $\mathrm{NH}_{2}$ deformation vibration; however, it was weakened or even disappeared in the spectra of QCS and LA-g-QC, and another peak at $1544 \mathrm{~cm}^{-1}$ corresponding to N-H and C-N of amido bond 
TABLE 1: Chemical compositions and properties of polymer micelles $(n=3)$.

\begin{tabular}{lcccc}
\hline Sample & $N(\mathrm{C}) / N(\mathrm{O})$ & Standard deviation $(\%)$ & Degree of quaternization (\%) & Degree of substitution (\%) \\
\hline CS & 1.13 & 0.3 & - & - \\
QCS & 1.41 & 2.3 & 42.6 & - \\
LA-g-QC & 2.05 & 1.6 & - & 27.4 \\
\hline
\end{tabular}

Note. $N(\mathrm{C}) / N(\mathrm{O})$ : the rate of the number of carbon elements to the number of oxygen elements in polymer. The table showed the degree of quaternization of the QCS and the degree of substitution of LA-g-QC through the elemental analysis.

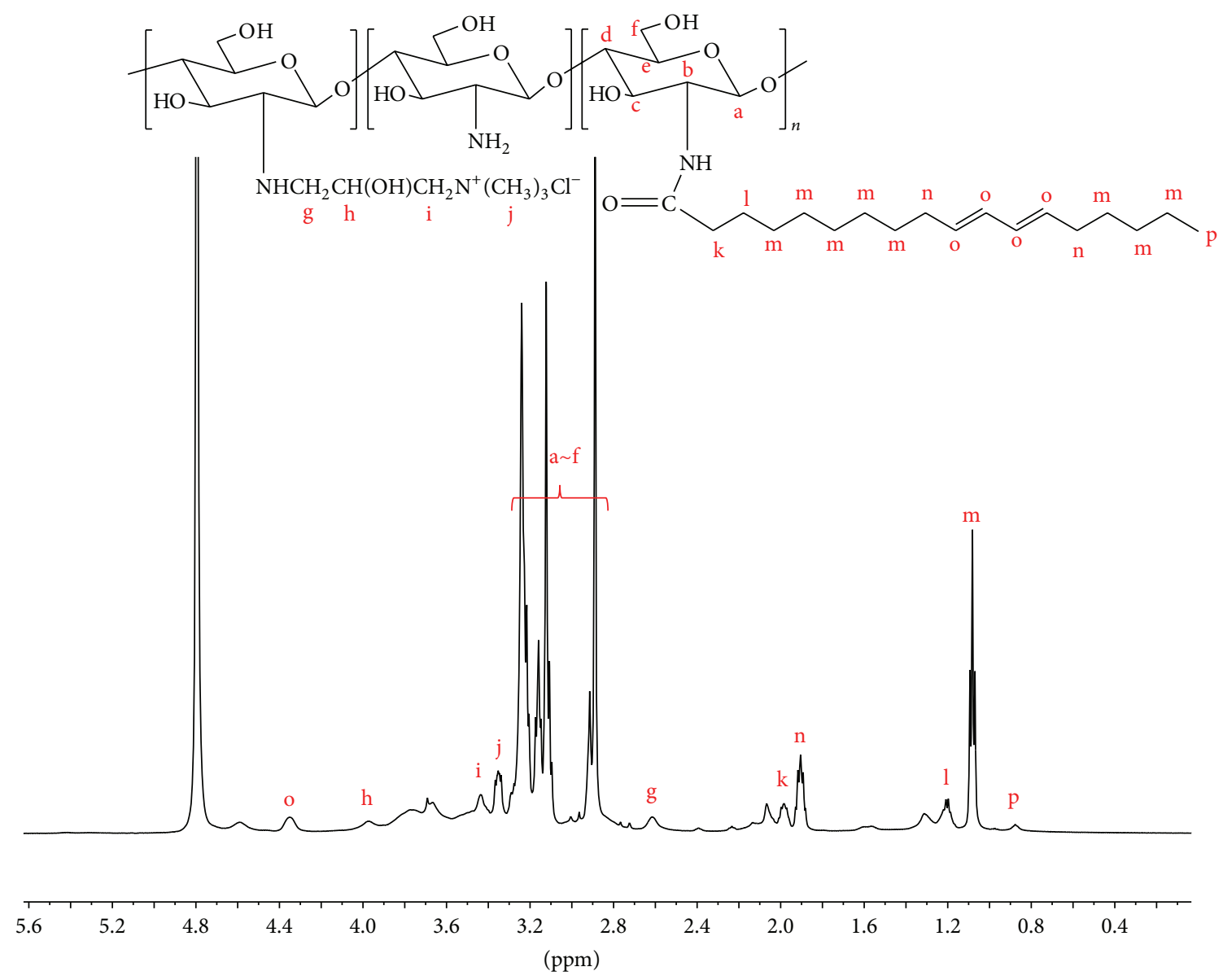

Figure $3:{ }^{1} \mathrm{H}-\mathrm{NMR}$ spectra of LA-g-QC in $\mathrm{D}_{2} \mathrm{O}$.

appeared. Additionally, a new peak at $1483 \mathrm{~cm}^{-1}$ emerged due to the methyl groups in the quaternary ammonium salt [15]. Interestingly, the characteristic peaks of primary alcohol and secondary alcohol in QCS samples positioned between $1152 \mathrm{~cm}^{-1}$ and $1030 \mathrm{~cm}^{-1}$ were consistent with the ones in original chitosan. The results indicated that the $\mathrm{N}$ monosubstitution on $\mathrm{C}_{2}$ position of chitosan was modified by introducing the ETA as expected. Moreover, in the spectra of LA-g-QC, another new peak at $1750 \mathrm{~cm}^{-1}$ appeared due to the ester groups, proving that the LA was successfully grafted into the quaternary chitosan. In order to confirm the successful synthesis of LA-g-QC, ${ }^{1} \mathrm{H}-\mathrm{NMR}$ spectra of LA-g-QC are shown in Figure 3. Besides the typical signals for chitosan, including $\delta=2.87 \sim 3.15 \mathrm{ppm}$ for $\mathrm{CH}$ groups and $\delta=3.23 \mathrm{ppm}$ for $\mathrm{CH}_{2}$, it can be observed at $\delta=2.61$, 3.44 ppm for $\mathrm{CH}_{2}$ groups, $\delta=3.35 \mathrm{ppm}$ for $\mathrm{CH}_{3}$ groups, and $\delta=3.97 \mathrm{ppm}$ for $\mathrm{CH}$ groups of quaternary ammonium salt. Last but not least, the signals were detected at $\delta=4.35 \mathrm{ppm}$ and $\delta=0.87 \sim 1.98 \mathrm{ppm}$ for LA. These results suggested that quaternary ammonium salt and LA were successfully grafted onto chitosan.

3.2. Elemental Analysis. The substitution degree (SD) of quaternary ammonium salt and LA was important parameter for LA-g-QC. According to the elemental analysis, the substitution degree was calculated based on the $\mathrm{C}$ and $\mathrm{N}$ molar ratios. It can be seen from Table 1 that the standard deviation of the prepared polymers QCS and LA-g-QC was between $2.3 \%$ and $1.6 \%$, respectively. The substitution degree (SD) of quaternary ammonium salt and LA was $42.6 \%$ and $27.4 \%$, respectively. 


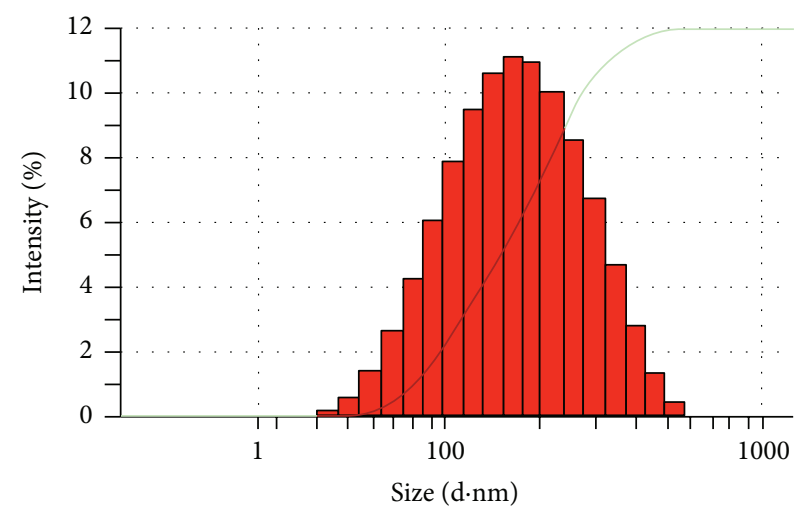

(a)

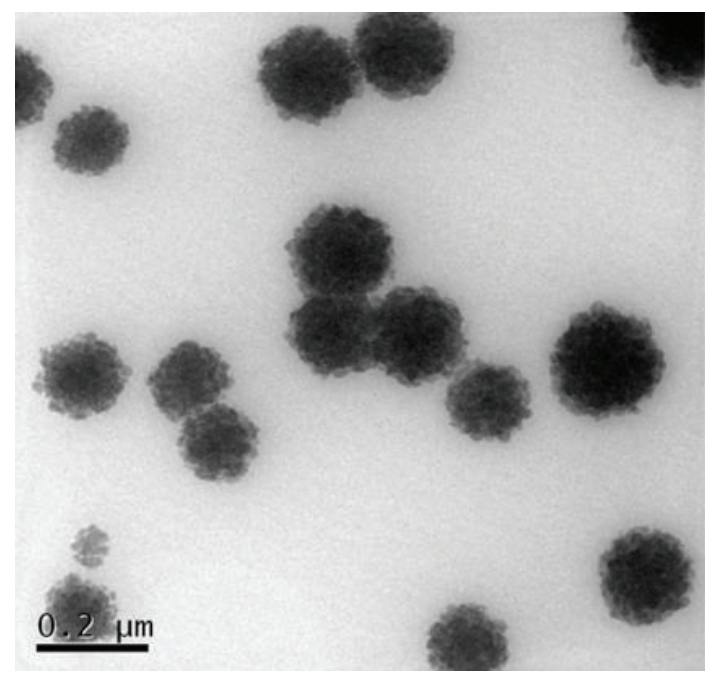

(c)

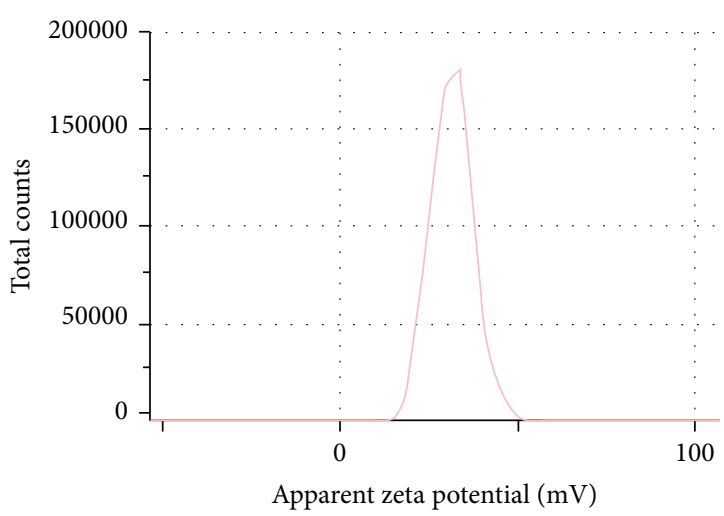

(b)

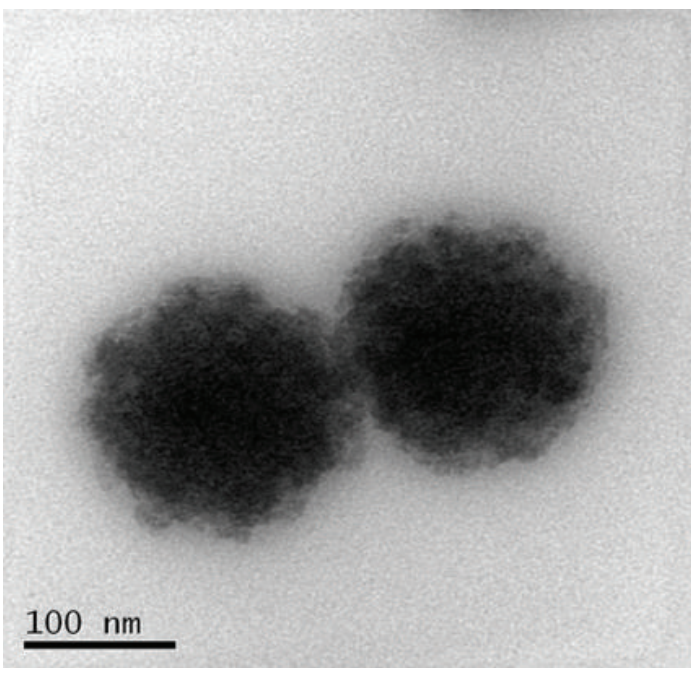

(d)

FIgure 4: (a) The average particle size of LA-g-QC micelles was $136.5 \mathrm{~nm}$ with a narrow distribution. (b) The zeta potential of LA-g-QC micelles. It showed that the zeta potential was about $+17.4 \mathrm{mV}$. (c) and (d) The TEM images of LA-g-QC micelles. It showed that the micelles had a spherical shape and the size of the micelles was slightly smaller than that determined by the DLS.

3.3. Particle Size and Zeta Potential. The particle size and zeta potential of LA-g-QC were analyzed, respectively. Figure 4(a) showed that LA-g-QC had a narrow distribution (PDI = $0.201,<0.5=$ with an average size of $136.5 \mathrm{~nm}$ ). Figure $4(\mathrm{~b})$ indicated that the zeta potential was approximately $+17.4 \mathrm{mV}$. Both results proved that LA-g-QC exhibited good properties as micelles. As reported, small nanocarriers $(<200 \mathrm{~nm})$ with positive charge can easily prevent the reticuloendothelial cell systems from uptake while tending to combine with the negative charge in cellular membrane. Therefore, the bioavailability of bioactive drugs can be enhanced after encapsulation into LA-g-QC.

3.4. Transmission Electron Microscopy (TEM). The TEM images of LA-g-QC micelles were shown in Figure 4(c), illustrating that the micelles had homogeneous spherical shapes and smooth surfaces without cracks. Micelles size viewed from TEM seemed to be slightly smaller compared with those obtained from DLS determination, indicating that the hydrophilic backbone in the micelles might shrink while drying the TEM samples.

3.5. Critical Micelle Concentration (CMC). As CMC is an important characteristic to evaluate the aggregation properties of LA-g-QC, it was determined by using the probe fluorescence technique. Pyrene was applied as the fluorescence probe, which hardly solubilized in water but had a considerably high solubility in the hydrophobic region of the micelles such as LA-g-QC solution with a series of concentrations from $1.0 \times 10^{-4}$ to $1.0 \mathrm{mg} / \mathrm{mL}$. The results demonstrated that the fluorescence intensity rose as the concentration of LA-g-QC increased. The fluorescence of pyrene in water is negligible because of the extremely low solubility. However, when the concentration was above $\mathrm{CMC}$, the solubility of pyrene would increase because the micelle core was formed as a subsequence, as a result of increased solution fluorescence. The fluorescence intensity ratio of pyrene $I_{3} / I_{1}(383 \mathrm{~nm} / 372 \mathrm{~nm})$ was analyzed as a 


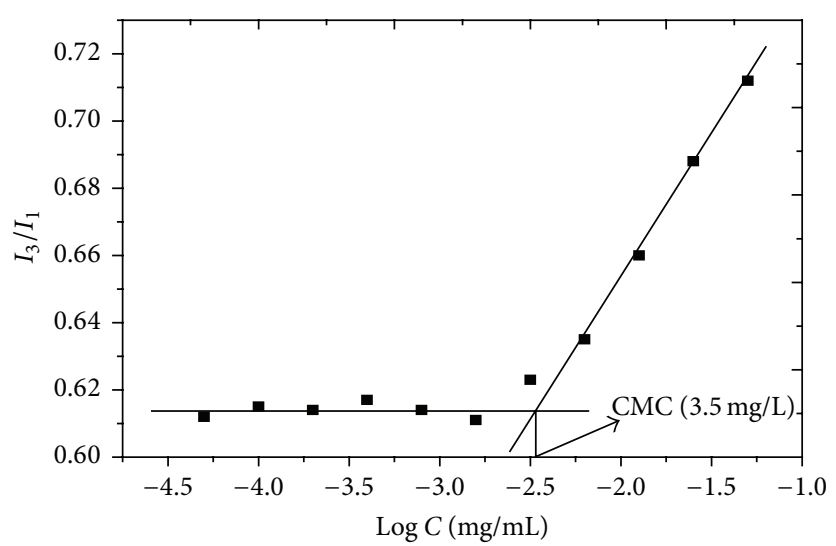

FIGURE 5: LA-g-QC micellar solution at different concentrations. The value of CMC was calculated as low as approximately $3.5 \mathrm{mg} / \mathrm{L}$.

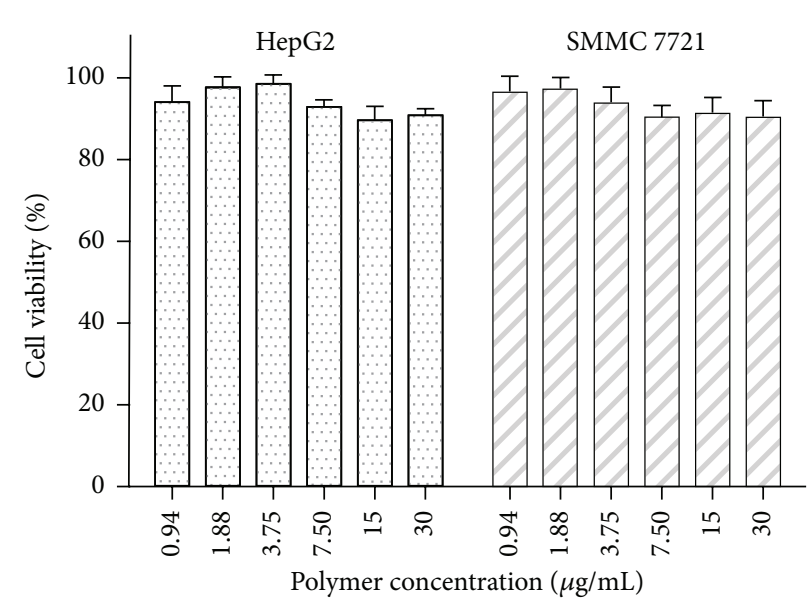

(a)

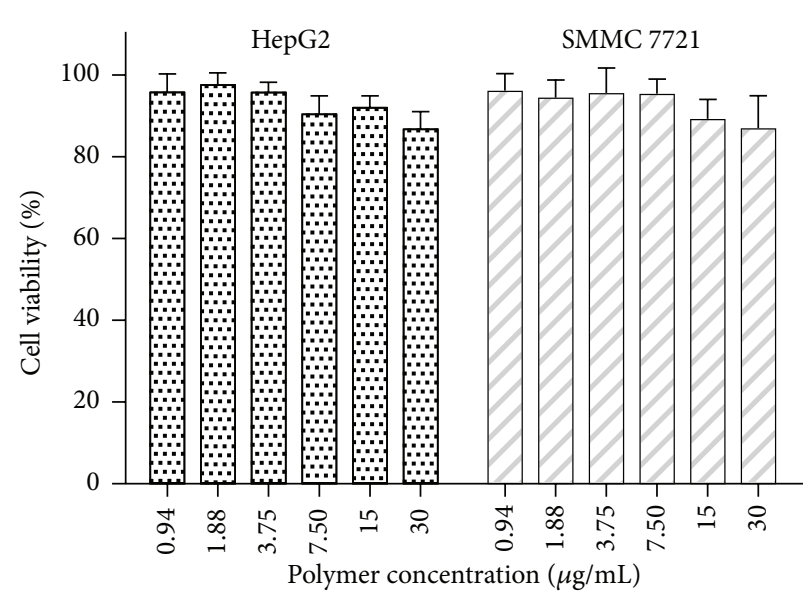

(b)

Figure 6: Cytotoxicity of LA-g-QC micelles against HepG2 and SMMC 7721 cells after $24 \mathrm{~h}$ (a) and $48 \mathrm{~h}$ (b) incubation.

logarithm function of the LA-g-QC concentration (Figure 5). A Boltzmann function was used to fit the scatters. By using the mathematical method, the value of CMC was calculated and shown as low as approximately $3.5 \mathrm{mg} / \mathrm{L}$. These results indicated that LA-g-QC was rational material for forming self-organized liposomes and might retain the integrity even upon strong dilution during systematic circulation.

3.6. In Vitro Cytotoxicity Assays. Highly biological safety should be the most important criterion for medical application. To figure out whether micelles constituted by LAg-QC copolymer have potential as drug carriers or probes, MTT assay has been applied to evaluate its cytotoxicity. HepG2 and SMMC 7721 cells were chosen to incubate with various concentrations of LA-g-QC micelles for $24 \mathrm{~h}$ and $48 \mathrm{~h}$, respectively. As shown in Figure 6, blank micelles did not significantly inhibit the cell proliferation, ranging from 0.94 to $30 \mu \mathrm{g} / \mathrm{mL}$ copolymer concentrations. After $24 \mathrm{~h}$ incubation, cell viability kept above $90 \%$. Even after incubation for $48 \mathrm{~h}$, low cytotoxicity of LA-g-QC copolymer was shown in vitro (Cell viability $>85 \%$ ). This result was consistent with the reported cytotoxicity of chitosan-based polymeric micelles, which showed that treatment with $15.6 \mu \mathrm{g} / \mathrm{mL}$ of hydrophobically modified sulfated chitosan for $48 \mathrm{~h}$ made approximately $90 \%$ of HepG 2 cells survive [18]. This result would provide evidences for the low biological toxicity and application potential of LA-g-QC micelles in biomedical field.

\section{Conclusion}

In this study, a novel amphiphilic chitosan derivative, LAg-QC copolymer, has been synthesized and characterized. The micelles are spheroids with a narrow size distribution of approximately $136.5 \mathrm{~nm}$. With a merit of low CMC comparing to other similar CMCs-based micelle systems, LA-g-QC has a high potential to form self-organized liposomes. In vitro study further indicated that the cytotoxicity of LA-g-QC copolymer was low, presenting a possibility in advancing the application of LA-g-QC copolymer. Collectively, the findings of our study suggest that LA-g-QC exhibits good properties as micelles.

\section{Conflict of Interests}

The authors declare that there is no conflict of interests regarding the publication of this paper. 


\section{Acknowledgments}

This study was supported by the Macao Science and Technology Development Fund (062/2013/A2), the Research Fund of the University of Macau (MYRG2014-00033-ICMSQRCM, MYRG2014-00051-ICMS-QRCM, MRG004/CMW/ 2014/ICMS, and MYRG2015-00171-ICMS-QRCM), and the National Natural Science Foundation of China (81403120 and 81473433).

\section{References}

[1] P. K. Dutta, J. Duta, and V. S. Tripathi, "Chitin and chitosan: chemistry, properties and applications," Journal of Scientific \& Industrial Research, vol. 63, no. 1, pp. 20-31, 2004.

[2] M. V. Dias, V. Machado Azevedo, S. V. Borges et al., "Development of chitosan/montmorillonite nanocomposites with encapsulated alpha-tocopherol," Food Chemistry, vol. 165, pp. 323329, 2014

[3] M. Rinaudo, "Chitin and chitosan: properties and applications," Progress in Polymer Science, vol. 31, no. 7, pp. 603-632, 2006.

[4] Y. Pranoto, S. K. Rakshit, and V. M. Salokhe, "Enhancing antimicrobial activity of chitosan films by incorporating garlic oil, potassium sorbate and nisin," LWT-Food Science and Technology, vol. 38, no. 8, pp. 859-865, 2005.

[5] A. Luzardo-Alvarez, N. Blarer, K. Peter et al., "Biodegradable microspheres alone do not stimulate murine macrophages in vitro, but prolong antigen presentation by macrophages in vitro and stimulate a solid immune response in mice," Journal of Controlled Release, vol. 109, no. 1-3, pp. 62-76, 2005.

[6] M. N. V. R. Kumar, R. A. A. Muzzarelli, C. Muzzarelli, H. Sashiwa, and A. J. Domb, "Chitosan chemistry and pharmaceutical perspectives," Chemical Reviews, vol. 104, no. 12, pp. 60176084, 2004

[7] A. Anitha, S. Sowmya, P. T. S. Kumar et al., "Chitin and chitosan in selected biomedical applications," Progress in Polymer Science, vol. 39, no. 9, pp. 1644-1667, 2014.

[8] M. E. I. Badawy, E. I. Rabea, and N. E. M. Taktak, "Antimicrobial and inhibitory enzyme activity of $\mathrm{N}$-(benzyl) and quaternary $\mathrm{N}$ (benzyl) chitosan derivatives on plant pathogens," Carbohydrate Polymers, vol. 111, pp. 670-682, 2014.

[9] J. Tong and L. G. Chen, "Review: preparation and application of magnetic chitosan derivatives in separation processes," Analytical Letters, vol. 46, no. 17, pp. 2635-2656, 2013.

[10] Y. Kakizawa and K. Kataoka, "Block copolymer micelles for delivery of gene and related compounds," Advanced Drug Delivery Reviews, vol. 54, no. 2, pp. 203-222, 2002.

[11] D. Q. Chen, P. Song, F. Jiang et al., "pH-responsive mechanism of a deoxycholic acid and folate comodified chitosan micelle under cancerous environment," The Journal of Physical Chemistry B, vol. 117, no. 5, pp. 1261-1268, 2013.

[12] H. Li and Z. M. Qian, "Transferrin/transferrin receptormediated drug delivery," Medicinal Research Reviews, vol. 22, no. 3, pp. 225-250, 2002.

[13] C. Dufes, J.-M. Muller, W. Couet, J.-C. Olivier, I. F. Uchegbu, and A. G. Schätzlein, "Anticancer drug delivery with transferrin targeted polymeric chitosan vesicles," Pharmaceutical Research, vol. 21, no. 1, pp. 101-107, 2004.

[14] L. Zavaleta-Avejar, E. Bosquez-Molina, M. Gimeno, J. P. PérezOrozco, and K. Shirai, "Rheological and antioxidant power studies of enzymatically grafted chitosan with a hydrophobic alkyl side chain," Food Hydrocolloids, vol. 39, pp. 113-119, 2014.

[15] J. W. Luo, X. Y. Wang, B. Xia, and J. Wu, "Preparation and characterization of quaternized chitosan under microwave irradiation," Journal of Macromolecular Science, Part A: Pure and Applied Chemistry, vol. 47, no. 9, pp. 952-956, 2010.

[16] X. Y. Wang, B. Liu, X. Li, and R. Sun, "Novel glucosamine hydrochloride-rectorite nanocomposites with antioxidant and anti-ultraviolet activity," Nanotechnology, vol. 23, no. 49, Article ID 495706, 2012.

[17] J. M. Zhang, Y. B. Li, W. Gao, M. A. Repka, Y. Wang, and M. Chen, "Andrographolide-loaded PLGA-PEG-PLGA micelles to improve its bioavailability and anticancer efficacy," Expert Opinion on Drug Delivery, vol. 11, no. 9, pp. 1367-1380, 2014.

[18] X.-H. Wang, Q. Tian, W. Wang, C.-N. Zhang, P. Wang, and Z. Yuan, "In vitro evaluation of polymeric micelles based on hydrophobically-modified sulfated chitosan as a carrier of doxorubicin," Journal of Materials Science: Materials in Medicine, vol. 23, no. 7, pp. 1663-1674, 2012. 

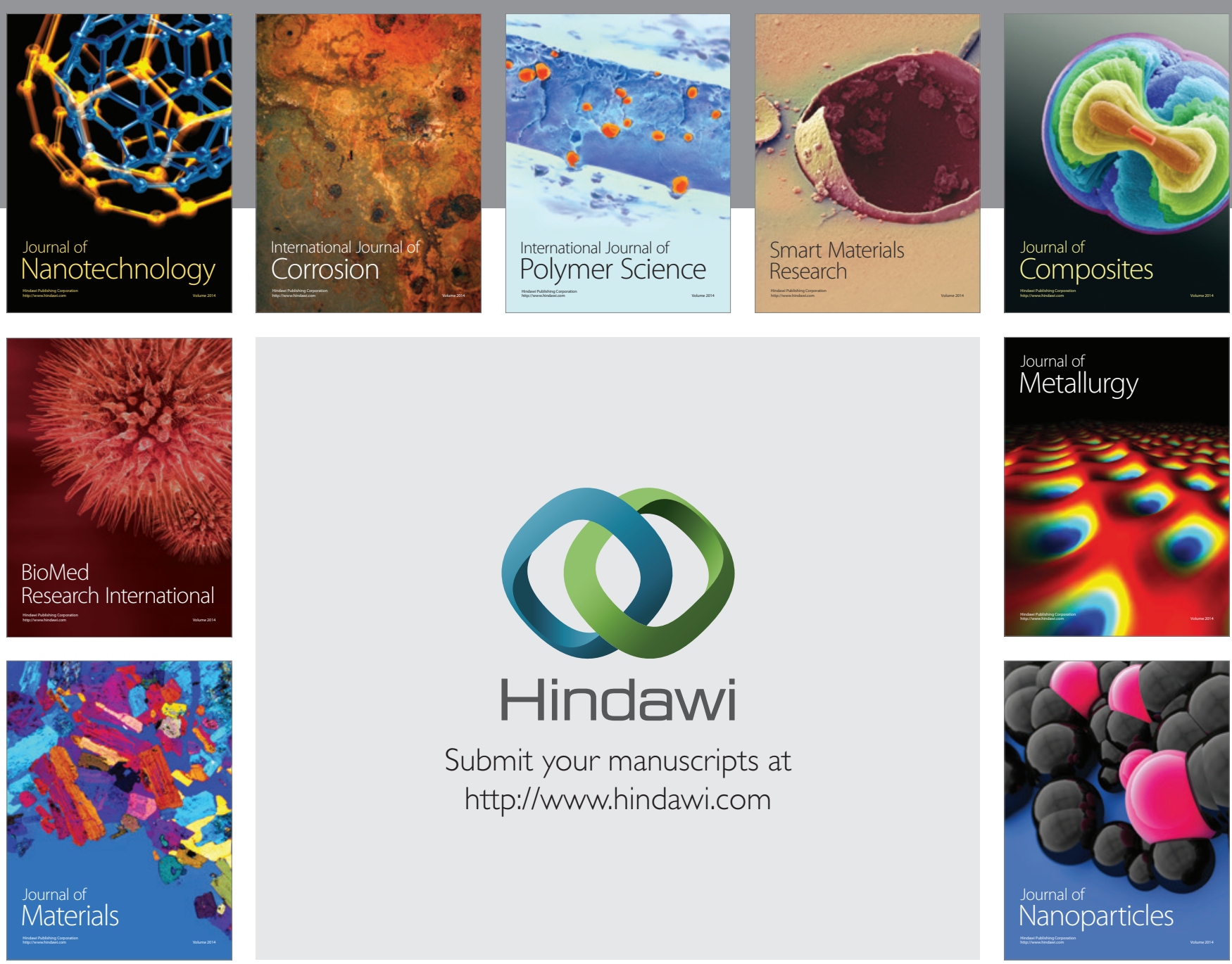

Submit your manuscripts at http://www.hindawi.com
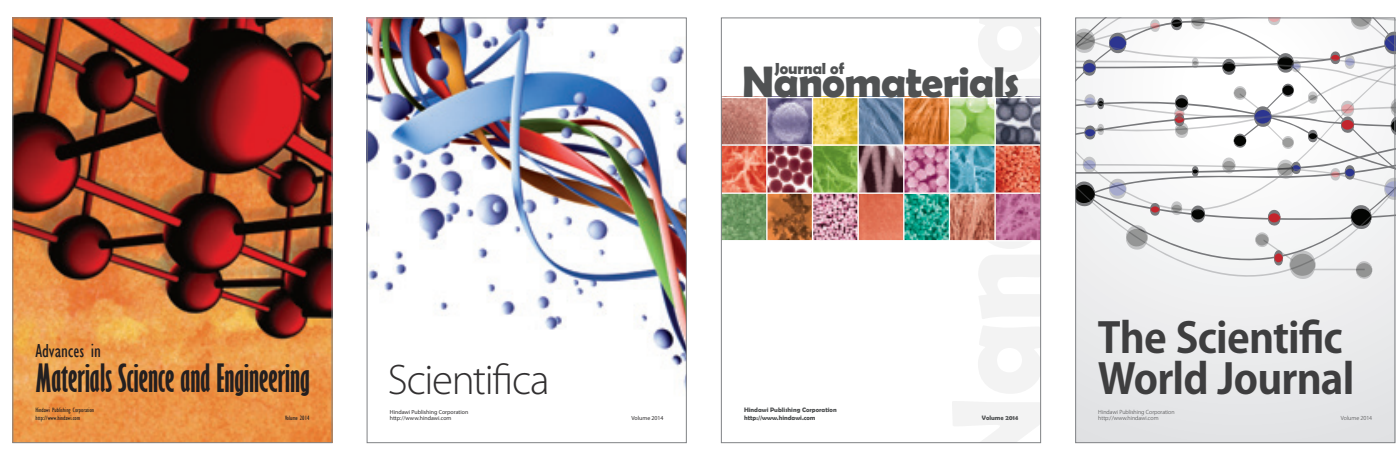

\section{The Scientific World Journal}
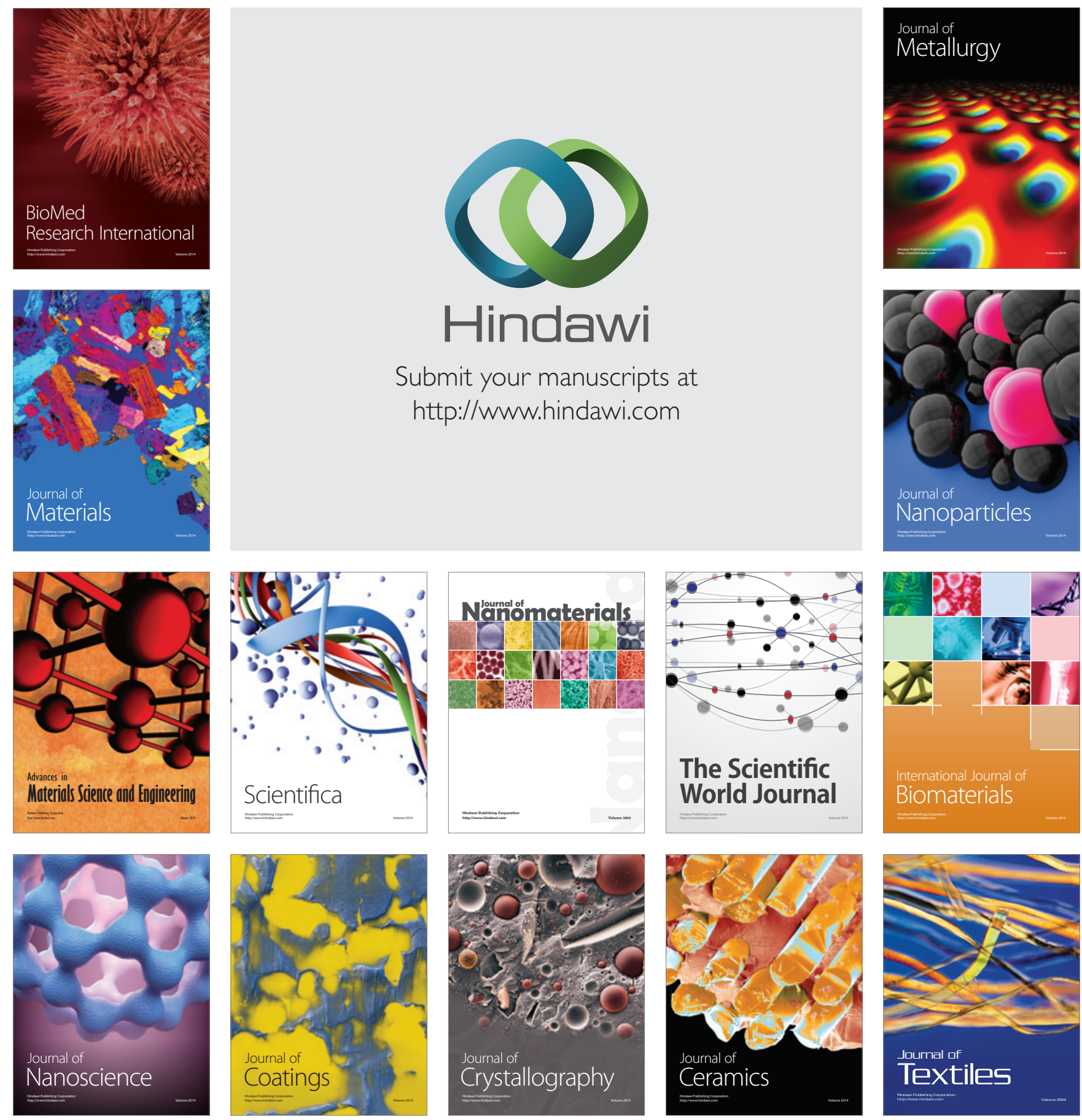